mus“ (S. 215). Infolgedessen erstreckt sich der Antiliberalismus auf die Linke wie die Rechte. So nannte schon de Gaulle Jean Monnet, immerhin der Vater des Plankommissariats, aber als Unternehmer auch jemand, der die Rolle des Marktes praktisch erfuhr und sie nicht in Frage stellte, einen „großen Amerikaner“ (was keineswegs als ein Kompliment gemeint war). Der ehemalige Premierminister Dominique de Villepin rief noch angesichts der drohenden Übernahme eines französischen Unternehmens durch ein US-amerikanisches pathetisch zum „Wirtschaftspatriotismus“ auf. Und Jacques Chirac verstieg sich gar zu einem Vergleich des Liberalismus mit dem Kommunismus, die beide „Perversionen des menschlichen Denkens" (S. 223) seien. Besonders augenfällig ist der Widerstand gegen den Liberalismus in der Sozialistischen Partei. Im Scheitern der beiden sozialliberalen Reformer, Jacques Delors und Michel Rocard, von Hayward kenntnisreich beschrieben, kann man einen aufschlussreichen Beleg dafür sehen. Eine Prognose wagt Hayward nicht, aber die Chancen, die Anwendung liberaler Normen und Praktiken durchzusetzen, hält er mit der Wahl Nicolas Sarkozys für so gut wie nie zuvor. Da es aber keine „umfassende ideologische Umkehr, sondern eine wohl kalkulierte und pragmatische Anpassung " an die liberalen Kräfte geben dürfte (S.228), werden traditionalistischer Diskurs und eine schon weiter fortgeschrittene liberale Praxis weiter „koexistieren“.

Es wäre reizvoll gewesen, die Aufsätze, die ein facettenreiches Bild geben, durch ein das politische System der V. Republik kritisch bilanzierendes Streitgespräch zwischen einem französischen und einem deutschen Wissenschaftler zu ergänzen, um zu erkennen, ob es unter den Politologen der beiden Länder noch national bedingte Unterschiede der Beurteilung gibt.

Auch dieser Band enthält abschließend einen Dokumentationsteil mit einer Chronik, sozioökonomischen Basisdaten, Wahlergebnissen und einer Bibliographie, der das Jahrbuch zu einem unentbehrlichen Nachschlagewerk für jeden Frankreichforscher macht.

Adolf Kimmel

\title{
Leistungsfähigkeit internationaler Institutionen
}

Hasenclever, Andreas, Klaus-Dieter Wolf und Michael Zürn (Hrsg.): Macht und Ohnmacht internationaler Institutionen. Festschrift für Volker Rittberger, Campus Verlag, Frankfurt am Main / New York 2007, 393 Seiten, € 39,90.

Die vorzustellende Aufsatzsammlung ist eine Festschrift zum 65. Geburtstag des Tübinger Friedens- und Konfliktforschers Volker Rittberger. Herausgegeben haben sie Andreas Hasenclever, Klaus-Dieter Wolf und Michael Zürn, die sich wie alle darin versammelten Autoren der „Tübinger Schule“ im Sinne von Rittbergers Forschung und Engagement verbunden fühlen. Das Buch wirkt wie ein Kaleidoskop zum gewählten Thema „Macht und Ohnmacht internationaler Institutionen“. Es ist in drei thematische Einheiten gegliedert, die sich mit (1) der Zukunft der transatlantischen Beziehungen, (2) Merkmalen für die Leistungsfähigkeit internationaler Institutionen und (3) friedens- und entwicklungspolitischen Leistungen internationaler Institutionen beschäftigen. Die meisten Aufsätze enthalten ei- 
nen Rekurs auf die innerdisziplinären Debatten der Internationalen Beziehungen, deren Ansätze und Theorien.

Einen gelungenen Überblick über den Stand der Forschung zum Thema gibt die Einleitung von Andreas Hasenclever und Peter Mayer. Neben der Kategorisierung von Institutionen, deren Entwicklungsgeschichte und aktuellen Trends werden Kriterien angeboten, um den Erfolg oder Misserfolg von internationalen Institutionen gemessen an ihrer Einflussmöglichkeit und Leistungsfähigkeit festzustellen. Mit der Zukunft der transatlantischen Beziehungen, die sie aktuell in der Krise sehen, setzen sich Michael Zürn und Martin Binder auseinander. Chancen zu ihrer Überwindung liegen in der gemeinsamen (allerdings zeitversetzten) Bedrohungswahrnehmung des internationalen Terrorismus, in einem gemeinsamen Wertekonsens, den ökonomischen wie kommunikativen Verflechtungen und in der identitären Bedeutung der NATO. Allerdings hängt die produktive Nutzung dieser Potenziale davon ab, ob die EU-Mitgliedstaaten eine klare Sicherheitsstrategie entwickeln und umsetzen, wozu nach Auffassung der Autoren der militärische Beitrag zur Lösung globaler Sicherheitsprobleme genauso gehört wie die Erschließung neuer Ansprechpartner in den USA. Sie bewerten diesen „formativen Prozess“ als offen. Wolfgang Wagner plädiert mit Blick auf die EU für ein europäisches Außenpolitikregime, das sich auf die Einführung qualifizierter Mehrheitsentscheidungen (QMV) stützt. Die Gemeinsame Außen- und Sicherheitspolitik der EU (GASP) könne ihre Leistungsfähigkeit, nämlich schnell auf internationale Krisen und Ereignisse zu reagieren, dann steigern, wenn sie auf eine Vergemeinschaftung nach dem Vorbild der europäischen Außenhandelspolitik verzichte. Zentral sind dabei die Einigung auf außenpolitische Positionen sowie deren Delegation an einen „Prinzipal“ als „Gesicht“ der gemeinsamen Politik. Der Autor hält aufgrund der laufenden Europäisierungsprozesse eine ausreichende politische Substanz für gegeben. Christoph Weller bietet das Modell der machiavellistischen Außenpolitik als Folie an, um die aktuellen USamerikanischen Sicherheitsstrategien zu erklären, die aus einem verstärkt unilateralen und militärischen Vorgehen bei gleichzeitiger manipulativer Öffentlichkeitsarbeit bestehen. Der Autor ist skeptisch, ob die amerikanische Außen- und Sicherheitspolitik durch internationale Institutionen zu bändigen ist. Das machiavellistische Modell bietet seiner Ansicht nach die Grundlage dazu, dass in Europa das amerikanische Denkmuster verstanden und in Folge entsprechend reagiert werden kann.

Im zweiten Teil überprüfen Carmen Huckel, Lothar Rieth und Melanie Zimmer am Beispiel von drei Public Private Partnerships (PPPs) deren Effektivität. Sie kommen zu dem Ergebnis, dass diese von zwei Faktoren abhängt: der Inklusivität und dem Institutionalisierungsgrad. Ersterer bezieht sich auf die Gleichberechtigung staatlicher und nichtstaatlicher Akteure, der zweite umfasst die Formalisierung von Entscheidungs-, Überwachungs- und Sanktionierungsprozessen. Je höher jeweils der Grad, desto höher auch die Leistungsfähigkeit der untersuchten PPPs. Thomas Conzelmann und Klaus-Dieter Wolf untersuchen ebenfalls an drei Fallstudien grenzüberschreitende privatwirtschaftliche Selbstregulierung. Diese stellt im internationalen System eine legitime Alternative zu den Steuerungsleistungen von staatlichen Akteuren dar, wenn sie ergänzend zur öffentlichen Regelsetzung und Gemeinwohlkriterien gedacht sowie in öffentliche Regulierungsstrukturen eingebettet ist. Am Beispiel von WTO und GATT betrachtet Bernhard Zangl, ob durch zunehmende Verrechtlichung die Effektivität internationaler Institutionen steigt. Er beantwortet die Frage positiv. Allerdings kann es zu einer „dialektische(n) Eigendynamik“ (S. 200) kommen, wenn für gefällte Entscheidungen in einem betroffenen Staat kein Rückhalt entsteht und sich die 
Legitimitätsfrage stellt. Der Autor weist darauf hin, dass beim Ausbau internationaler Rechtsherrschaft die internationalen Streitinstanzen darauf achten müssen, ob es sich um einen Interessen- oder Wertekonflikt handelt, weil sich je nach Konflikt eine andere Vorgehensweise empfiehlt. Anhand der Entwicklung der Handelsbeziehung zwischen USA und Japan belegt Hartwig Hummel, dass die offensive Nutzung des WTO-Streitschlichtungsverfahrens durch Japan in der Region als Vorbild dient. Ebenso unterstützt das Vorgehen der USA die Integration in der Region, weil sie Schiedssprüche gegen sich selbst gelten lassen, solange diese die Liberalisierung im Rahmen der WTO ergänzen und nicht ersetzen.

Der dritte Teil beginnt mit Helmut Breitmeier, der sich mit der Effektivität der Vereinten Nationen bei Konfliktprävention und -lösung auseinandersetzt. Er nimmt hierzu die Anzahl zwischenstaatlicher Kriege in den Blick, die durch das Agieren der UNO abgenommen haben - ein Trend, der in der Öffentlichkeit kaum wahrgenommen und in der Wissenschaft wenig diskutiert wird. Martin List untersucht die Wirkung des internationalen Nichtverbreitungsregimes für Kernwaffen (NPT-Regime) am Beispiel des Iran. Seine Bewertung fällt kritisch aus: Das NPT-Regime befindet sich in der Krise, weil letztendlich die Mittel fehlen, um einen Verzicht auch wirksam durchzusetzen. Für die Kernwaffenstaaten entsteht der Auftrag, die Weiterverbreitung zu verhindern und sich bei Vermeidungsstrategien zu einigen. Ihr Machtkalkül kann dieser Notwendigkeit entgegenstehen; der weitere Prozess ist offen. Andreas Hasenclever und Brigitte Weiffen verteidigen die These, dass internationale Institutionen wesentlich zur geringen Gewaltanfälligkeit zwischenstaatlicher Beziehungen beitragen. Besonders effektiv zeigen sich internationale Institutionen mit überwiegend demokratischer Mitgliedschaft. Ein Grund liegt in der intensiven Vernetzung mit ihrer Zivilgesellschaft und der daraus entstehenden Transparenz und Kontrolle, die eine Verlässlichkeit durch Einbindung entstehen lassen. Im letzten Beitrag beschäftigt sich Joachim Betz mit Macht und Ohnmacht von IWF und Weltbank. Sein Fazit lautet, dass sie die internationale Entwicklungsdebatte nachhaltig beeinflussen, gleichzeitig aber zunehmend Konkurrenz durch private Organisationen bekommen. In ihrer Gestaltungsmacht ragen sie zwar über andere Institutionen hinaus, zeichnen sich jedoch durch eine gewisse „Überdehnung“ aus.

Insgesamt ist die Aufsatzsammlung als Festschrift gelungen, weil die innerdisziplinäre Bandbreite an Forschungsgegenständen, Theorien und Positionierungen sichtbar wird und in der Zusammenschau eine Fundierung bestehender Forschungsansätze liefert. Einige Beiträge wie etwa die von Zürn und Binder oder Hasenclever, Mayer und Weiffen sind durch ihre klare Struktur und den erklärenden Duktus besonders informativ, plausibel und lesenswert. Bei anderen wiederum wie etwa bei Weller oder List entsteht der Eindruck, dass sich die differenzierte Bearbeitung ihrer komplexen Fragestellungen dem Format eines Aufsatzes etwas entzieht. Wünschenswert wäre eine zusammenfassende oder zugespitzte Bewertung der Herausgeber am Ende des Bandes gewesen, um die Beiträge mit einer Klammer zu versehen. An einigen Stellen wirft die umständliche politikwissenschaftliche Terminologie die Frage nach der Leserschaft und Wirkung des Buches auf: innerdisziplinär ohne Frage lesenswert, mit dem Blick aus der politischen Praxis bleibt jedoch der Wunsch nach Erkenntnisgewinn für politisches Handeln eher unerfüllt. 